PROCEEDINGS OF THE

AMERICAN MATHEMATICAL SOCIETY

Volume 137, Number 3, March 2009, Pages 1127-1133

S 0002-9939(08)09618-4

Article electronically published on October 1, 2008

\title{
ABSTRACT ELEMENTARY CLASSES INDUCED BY TILTING AND COTILTING MODULES HAVE FINITE CHARACTER
}

\author{
JAN TRLIFAJ
}

(Communicated by Julia Knight)

\begin{abstract}
Let $R$ be a ring and $\mathcal{C}$ be a cotilting class of $R$-modules. Define $A \leq B$ by $A \subseteq B$ and $A, B, B / A \in \mathcal{C}$. Then $(\mathcal{C}, \leq)$ is an abstract elementary class of finite character. An analogous result holds for all abstract elementary classes induced by tilting modules.
\end{abstract}

\section{INTRODUCTION}

A surprising correspondence between algebraic properties of classes $\mathcal{C}$ studied in infinite dimensional tilting theory and model theoretic properties of the induced abstract elementary classes $(\mathcal{C}, \leq)$ has recently been discovered in $[3$. In this paper, we show that two important instances of this correspondence, namely the case when $\mathcal{C}$ is a cotilting class and when $\mathcal{C}={ }^{\perp} \mathcal{T}$ for a tilting class $\mathcal{T}$, always yield abstract elementary classes of finite character. The latter classes admit the tools of infinitary logics developed in [2, 9], and [10.

The notion of an abstract elementary class (or AEC, for short) goes back to Shelah [12] and provides a general framework for far reaching extensions of classical model theory of first order structures; cf. 2]. AECs of finite character were introduced by Hyttinen and Kesälä in [9]; recently, Kueker [10] has shown that an AEC $(\mathcal{C}, \leq)$ with amalgamation property has finite character if and only if $A \leq B$ whenever $A, B \in \mathcal{C}$ are such that $A \subseteq B$, and for each finite subset $X$ of $A$ there is a monomorphism $f_{X}: A \rightarrow B$ with $f_{X}\left\lceil X=\operatorname{id}_{X}\right.$ and $f_{X}(A) \leq B$.

In 3 particular AECs were introduced, namely those of the form $\left({ }^{\perp} \mathcal{D}, \leq\right)$ where $\mathcal{D}$ is a class of modules over an associative ring $R$,

$$
{ }^{\perp} \mathcal{D}=\bigcap_{1 \leq i<\omega} \operatorname{KerExt}_{R}^{i}(-, \mathcal{D})=\left\{M \mid \operatorname{Ext}_{R}^{i}(M, D)=0 \text { for all } D \in \mathcal{D} \text { and } i \geq 1\right\}
$$

and $A \leq B$ means that $A$ is a submodule of $B$ such that $A, B, B / A \in{ }^{\perp} \mathcal{D}$. Since these AECs have the amalgamation property by [3, Lemma 2.1], we will take Kueker's characterization above as our definition of finite character.

In 3 a question was raised as to whether the AECs of the form $\left({ }^{\perp} \mathcal{D}, \leq\right)$ have finite character; cf. 3, Question 4.1.(2)].

Received by the editors December 14, 2007, and, in revised form, April 15, 2008.

2000 Mathematics Subject Classification. Primary 03C95, 16E30; Secondary 03C60, 16D90.

Key words and phrases. Abstract elementary class of finite character, definable class, tilting module, cotilting module, Ext.

This research was supported by GAČR 201/06/0510 and MSM 0021620839.

(C)2008 American Mathematical Society Reverts to public domain 28 years from publication 
Our main result here gives a positive answer in the case when ${ }^{\perp} \mathcal{D}$ is a cotilting class of modules over an arbitrary ring. It follows that all AECs of the form $\left({ }^{\perp} \mathcal{D}, \leq\right)$ over a Dedekind domain, or a right artinian right hereditary ring, have finite character. We also prove that if $\mathcal{T}$ is a tilting class over an arbitrary ring and $\left({ }^{\perp} \mathcal{T}, \leq\right)$ is an AEC, then $\left({ }^{\perp} \mathcal{T}, \leq\right)$ has finite character.

Recall that a pair $(\mathcal{C}, \leq)$ is an abstract elementary class if $\mathcal{C}$ is a class of $\tau$ structures (for some vocabulary $\tau$ ) and $\leq$ is a partial order on $\mathcal{C}$, and both $\mathcal{C}$ and $\leq$ are closed under isomorphism and satisfy:

- (A1) If $A \leq B$, then $A$ is a substructure of $B$.

- (A2) If $\left(A_{i} \mid i<\delta\right)$ is a $\leq$-increasing chain of elements of $\mathcal{C}$ (that is, $A_{i} \leq A_{i+1}$ for all $i<\delta$, and $A_{i}=\bigcup_{j<i} A_{j}$ for all limit ordinals $i<\delta$ ), then

(1) $\bigcup_{i<\delta} A_{i} \in \mathcal{C}$

(2) $A_{j} \leq \bigcup_{i<\delta} A_{i}$ for each $j<\delta$;

(3) if $M \in \mathcal{C}$ and $A_{i} \leq M \in \mathcal{C}$ for each $j<\delta$, then $\bigcup_{i<\delta} A_{i} \leq M$.

- (A3) If $A, B, C \in \mathcal{C}, \bar{A} \leq C, B \leq C$ and $A$ is a substructure of $B$, then $A \leq B$.

- (A $\overline{4})$ There is a "Löwenheim-Skolem" cardinal number $\kappa$ such that if $A$ is a substructure of $B \in \mathcal{C}$, then there is $A^{\prime} \in \mathcal{C}$ which contains $A$ as a substructure so that $A^{\prime} \leq B$, and the cardinality of $A^{\prime}$ is at most $|A|+\kappa$.

If $A \leq B$, then we will say that $A$ is strong in $B$.

The basic example of an AEC is the class of all models of a first order theory with the relation of being an elementary submodel. The examples relevant here have the form $\left({ }^{\perp} \mathcal{D}, \leq\right)$ for a class of right $R$-modules $\mathcal{D}$ with the relation $A \leq B$ if $A$ is a submodule of $B$ such that $A, B, B / A \in{ }^{\perp} \mathcal{D}$.

We have no restriction on the cardinality of the vocabulary: our running convention here is that ' $\tau$-structure' stands for 'right $R$-module' over an arbitrary, but fixed, ring $R$, and 'substructure' stands for 'submodule'. Then $\left({ }^{\perp} \mathcal{D}, \leq\right)$ is an AEC if and only if the class ${ }^{\perp} \mathcal{D}$ is closed under direct limits and has refinements (see Lemma 2.1 below).

These two properties of ${ }^{\perp} \mathcal{D}$ are not easy to check. However, there is an important case where they always hold: by 3. Theorem 0.1(1)] and [14, Theorem 13], this happens when $\mathcal{C}={ }^{\perp} \mathcal{D}$ is a cotilting class, that is, when $\mathcal{C}={ }^{\perp}\{C\}$ for a cotilting module $C$.

Recall that a module $C$ is cotilting provided that

- (C1) $C$ has finite injective dimension.

- (C2) $\operatorname{Ext}_{R}^{i}\left(C^{I}, C\right)=0$ for each $i>0$ and each set $I$.

- (C3) There are an injective cogenerator $W$ for Mod- $R$ and a finite exact sequence $0 \rightarrow C_{n} \rightarrow \cdots \rightarrow C_{0} \rightarrow W \rightarrow 0$, where $C_{i}$ a direct summand of a direct product of copies of the module $C$ for each $i \leq n$.

For example, if $R$ is a Dedekind domain, then the AECs of the form $\left({ }^{\perp} \mathcal{D}, \leq\right)$ for a class of modules $\mathcal{D}$ are exactly the $\operatorname{AECs}(\mathcal{C}, \leq)$ where $\mathcal{C}$ is a cotilting class, and they correspond bijectively to subsets $P$ of the maximal spectrum of $R$. The class $\mathcal{C}$ corresponding to $P$ is the class of all modules that are $p$-torsion free for each $p \in P$ (see [3], Theorem $0.2(3)]$ ).

The notion of a cotilting module is a formal dual of the better known notion of an (infinitely generated) tilting module where a module $T$ is said to be tilting provided that 
- (T1) $T$ has finite projective dimension.

- (T2) $\operatorname{Ext}_{R}^{i}\left(T, T^{(I)}\right)=0$ for each $i>0$ and each set $I$.

- (T3) There is a finite exact sequence $0 \rightarrow R \rightarrow T_{0} \rightarrow \cdots \rightarrow T_{n} \rightarrow 0$, where $T_{i}$ a direct summand in a direct sum of copies of the module $T$ for each $i \leq n$.

If $T$ is a tilting module, then

$$
\{T\}^{\perp}=\bigcap_{1 \leq i<\omega} \operatorname{KerExt}_{R}^{i}(T,-)=\left\{M \mid \operatorname{Ext}_{R}^{i}(T, M)=0 \text { for all } i \geq 1\right\}
$$

is the tilting class induced by $T$.

Given a ring $R$ we let Mod- $R$ denote the category of all right $R$-modules and $\bmod -R$ the subcategory of all strongly finitely presented modules, that is, of the modules possessing a (possibly infinite) projective resolution consisting of finitely generated modules.

A class $\mathcal{C} \subseteq \operatorname{Mod}-R$ is resolving provided that $R \in \mathcal{C}, \mathcal{C}$ is closed under extensions and direct summands, and $C_{1} \in \mathcal{C}$ whenever there is a short exact sequence of the form $0 \rightarrow C_{1} \rightarrow C_{2} \rightarrow C_{3} \rightarrow 0$, where $C_{2}, C_{3} \in \mathcal{C}$. For example, mod $-R$ is resolving.

A class $\mathcal{D} \subseteq \operatorname{Mod}-R$ is said to be of finite type in the case where there is a class $\mathcal{S} \subseteq \bmod -R$ such that $\mathcal{D}=\mathcal{S}^{\perp}=\bigcap_{1 \leq i<\omega} \operatorname{KerExt}_{R}^{i}(\mathcal{S},-)$. Moreover, if all modules in $\mathcal{S}$ have bounded projective dimension, then $\mathcal{D}$ is said to be of bounded type. In this notation, the main result of [6] says that tilting classes coincide with the classes of bounded type.

We refer to [2, 3] and [8] for basic properties of the notions defined above 1 We only note that in the general setting of infinitely generated modules, cotilting theory amounts to more than just dualization of the (infinite dimensional) tilting theory: while the dual (character module) of any tilting left $R$-module is a cotilting right $R$-module, Bazzoni has recently discovered a class of valuation domains with cotilting modules that are not equivalent to duals of any tilting modules, 4.

\section{AECs INDUCED By COTILTING MODUleS}

In this section we will consider the AECs of the form $(\mathcal{C}, \leq)$, where $\mathcal{C}$ is a cotilting class of modules, and show that they are of finite character. We will see that $\mathcal{C}$ is always axiomatizable in the language of the first order theory of modules, so the complexity of this case rests in the unusual relation of $\leq$.

We start by recalling a well-known tensor vanishing criterion:

Lemma 1.1. Let $R$ be a ring, $D$ be a left $R$-module with a finite generating set $\left(d_{i} \mid i<m\right), C$ be a right $R$-module, and $\left\{c_{i} \mid i<m\right\}$ be a sequence of elements of $C$. Then the following are equivalent:

(1) $\sum_{i<m} c_{i} \otimes d_{i}=0$ in $C \otimes_{R} D$.

(2) There exist $0<n<\omega$, a matrix $A=\left(a_{j i}\right) \in M_{n \times m}(R)$ and elements $\left(c_{j}^{\prime} \mid\right.$ $j<n)$ in $C$ such that $\sum_{i<m} a_{j i} d_{i}=0$ for each $j<n$, and $c_{i}=\sum_{j<n} c_{j}^{\prime} a_{j i}$ for each $i<m$.

Proof. This is a particular instance of [13, Proposition I.8.8].

A class of modules $\mathcal{C}$ is said to be definable provided that $\mathcal{C}$ is closed under direct products, direct limits, and pure submodules.

\footnotetext{
${ }^{1}$ However, for simplicity of notation, we occasionally deviate from the notation therein.
} 
There are many characterizations of definable classes of modules; cf. [7, 2.3]. In particular, each definable class is axiomatizable in the language of the first order theory of modules by axioms saying that certain Baur-Garavaglia-Monk invariants are 1; see [11]. We will need the following characterization due to Bazzoni (see [5, Proposition 5.2]):

Lemma 1.2. Let $R$ be a ring and $\mathcal{C}$ be a class of right $R$-modules. Then the following are equivalent:

(1) $\mathcal{C}$ is definable.

(2) There is a set of $R$-homomorphisms $\Theta=\left\{\theta_{i}: F_{i} \rightarrow G_{i} \mid i \in I\right\}$ with all $F_{i}$ 's and $G_{i}$ 's finitely presented left $R$-modules, such that $\mathcal{C}=\operatorname{Ker}(\Theta)$ where

$\operatorname{Ker}(\Theta)=\left\{M \in \operatorname{Mod}-R \mid i d_{M} \otimes_{R} \theta_{i}: M \otimes_{R} F_{i} \rightarrow M \otimes_{R} G_{i}\right.$ is monic for all $\left.i \in I\right\}$.

Let $\mathcal{C}$ be a class of modules. Then $\mathcal{C}$ is special provided that for each module $B$ there exists an exact sequence $0 \rightarrow B \rightarrow D \rightarrow C \rightarrow 0$ with $C \in \mathcal{C}$ and $D \in \mathcal{C}^{\perp}$.

Cotilting classes are particular sorts of definable classes:

Lemma 1.3. Let $R$ be a ring and $\mathcal{C}$ be a cotilting class of right $R$-modules. Then $\mathcal{C}$ is definable, special, and resolving.

Proof. This follows e.g. from [8, Theorems 4.3.23 and 8.1.10].

Notice that if $\mathcal{C}$ is cotilting, then any set $\Theta$ such that $\mathcal{C}=\operatorname{Ker}(\Theta)$ as in Lemma 1.2(2) must consist of monomorphisms, since $R \in \mathcal{C}$.

Theorem 1.4. Let $R$ be a ring and $\mathcal{C}$ be a cotilting class of right $R$-modules. Then $(\mathcal{C}, \leq)$ is an $A E C$ of finite character.

Proof. Assume that $(\mathcal{C}, \leq)$ is not of finite character. Then there are modules $A \subseteq B \in \mathcal{C}$ such that $B / A \notin \mathcal{C}$, but for each finite subset $X \subseteq A$ there is a monomorphism $f_{X}: A \rightarrow B$ with $f_{X}\left\lceil X=\operatorname{id}{ }_{X}\right.$ and $B / f_{X}(A) \in \mathcal{C}$.

By Lemma 1.3 $\mathcal{C}$ is special, so there is an exact sequence $0 \rightarrow B \subseteq D \rightarrow C \rightarrow 0$ with $D \in \mathcal{C}^{\perp}$ and $B, C, D \in \mathcal{C}$, and $\mathcal{C}$ is resolving, so $D / A \notin \mathcal{C}$.

By Lemmas 1.3 and 1.2, there is a set of $R$-monomorphisms $\Theta$ such that $\mathcal{C}=$ $\operatorname{Ker} \Theta$. Since $D / A \notin \mathcal{C}$ there exist finitely presented left $R$-modules $F \subseteq G$ such that the inclusion $\theta: F \hookrightarrow G$ belongs to $\Theta$ but the map $i d_{D / A} \otimes_{R} \theta:(D / A) \otimes_{R} F \rightarrow$ $(D / A) \otimes_{R} G$ is not monic.

Let $\left\{f_{i} \mid i<m\right\}$ be an $R$-generating subset of $F$, and $\left\{g_{j} \mid j<n\right\}$ an $R_{-}$ generating subset of $G$. Then there is a matrix $\left(r_{i j}\right) \in M_{m \times n}(R)$ such that $f_{i}=$ $\sum_{j<n} r_{i j} g_{j}$ for each $i<m$. By assumption there exist elements $\left(d_{i} \mid i<m\right)$ in $D$ such that $\sum_{i<m}\left(d_{i}+A\right) \otimes_{R} f_{i} \neq 0$ in $(D / A) \otimes_{R} F$ but $\sum_{i<m}\left(d_{i}+A\right) \otimes_{R} f_{i}=$ $\sum_{j<n}\left(\sum_{i<m} d_{i} r_{i j}+A\right) \otimes_{R} g_{j}=0$ in $(D / A) \otimes_{R} G$.

By Lemma 1.1, the latter just says that there exist elements $\left(d_{k}^{\prime} \mid k<p\right)$ in $D$ and a matrix $\left(s_{k j}\right) \in M_{p \times n}(R)$ such that $\sum_{i<m} s_{k j} g_{j}=0$ for each $k<p$ and $\left(\sum_{i<m} d_{i} r_{i j}\right)+A=\left(\sum_{k<p} d_{k}^{\prime} s_{k j}\right)+A$ for each $j<n$. In particular, we can define $\left(a_{j} \mid j<n\right)$ in $A$ by $a_{j}=\left(\sum_{i<m} d_{i} r_{i j}\right)-\left(\sum_{k<p} d_{k}^{\prime} s_{k j}\right)$ for each $j<n$. Then $\sum_{j<n}\left(\sum_{i<m} d_{i} r_{i j}-a_{j}\right) \otimes_{R} g_{j}=0$ in $D \otimes_{R} G$ by Lemma 1.1

Take $X=\left\{a_{j} \mid j<n\right\}$ and define $I=f_{X}(A)$. Since $a_{j}=f_{X}\left(a_{j}\right)$ for each $j<n$, Lemma 1.1 implies that $\sum_{j<n}\left(\sum_{i<m} d_{i} r_{i j}+I\right) \otimes_{R} g_{j}=0$ in $(D / I) \otimes_{R} G$. Since $B / I \in \mathcal{C}$ and $(D / I) /(B / I) \cong C \in \mathcal{C}$, also $D / I \in \mathcal{C}$, so the map $i d_{D / I} \otimes_{R} \theta$ : $(D / I) \otimes_{R} F \rightarrow(D / I) \otimes_{R} G$ is monic; thus $\sum_{i<m}\left(d_{i}+I\right) \otimes_{R} f_{i}=0$ in $(D / I) \otimes_{R} F$. 
Denote by $\nu$ the inclusion $\nu: I \hookrightarrow D$. Then $\sum_{i<m} d_{i} \otimes_{R} f_{i} \in \operatorname{Im}\left(\nu \otimes_{R} \operatorname{id}_{F}\right) \subseteq$ $D \otimes_{R} F$, so there exist elements $\left(a_{i}^{\prime} \mid i<m\right)$ in $A$ such that $\sum_{i<m}\left(d_{i}-f_{X}\left(a_{i}^{\prime}\right)\right) \otimes_{R}$ $f_{i}=0$ in $D \otimes_{R} F$.

It follows that $\sum_{i<m}\left(d_{i}-f_{X}\left(a_{i}^{\prime}\right)\right) \otimes_{R} f_{i}=\sum_{j<n}\left(\sum_{i<m}\left(d_{i}-f_{X}\left(a_{i}^{\prime}\right)\right) r_{i j}\right) \otimes_{R} g_{j}=0$ in $D \otimes_{R} G$. By Lemma 1.1 there exist elements $\left(\bar{b}_{l} \mid l<q\right)$ in $D$ and a matrix $\left(t_{l j}\right) \in$ $M_{q \times n}(R)$ such that $\sum_{j<n} t_{l j} g_{j}=0$ for each $l<q$, and $\sum_{i<m}\left(d_{i}-f_{X}\left(a_{i}^{\prime}\right)\right) r_{i j}=$ $\sum_{l<q} \bar{b}_{l} t_{l j}$ for each $j<n$. Then

$$
f_{X}\left(a_{j}\right)+\sum_{k<p} d_{k}^{\prime} s_{k j}=a_{j}+\sum_{k<p} d_{k}^{\prime} s_{k j}=\sum_{i<m} d_{i} r_{i j}=\sum_{i<m} f_{x}\left(a_{i}^{\prime}\right) r_{i j}+\sum_{l<q} \bar{b}_{l} t_{l j} .
$$

Therefore, $f_{X}\left(a_{j}-\sum_{i<m} a_{i}^{\prime} r_{i j}\right)=\sum_{l<q} \bar{b}_{l} t_{l j}-\sum_{k<p} d_{k}^{\prime} s_{k j}$ for each $j<n$, and Lemma 1.1 yields $\sum_{j<n} f_{X}\left(a_{j}-\sum_{i<m} a_{i}^{\prime} r_{i j}\right) \otimes g_{j}=0$ in $D \otimes_{R} G$.

Since $f_{X}$ is monic, there exists $g_{X}=f_{X}^{-1}: I \rightarrow D$ such that $g_{X} f_{X}=i d_{A}$. However, $D / I \in \mathcal{C}$ and $D \in \mathcal{C}^{\perp}$, so $g_{X}$ extends to an $R$-homomorphism $g: D \rightarrow D$. Then

$$
\left(g \otimes_{R} G\right)\left(\sum_{j<n} f_{X}\left(a_{j}-\sum_{i<m} a_{i}^{\prime} r_{i j}\right) \otimes g_{j}\right)=\sum_{j<n}\left(a_{j}-\sum_{i<m} a_{i}^{\prime} r_{i j}\right) \otimes g_{j}=0
$$

in $D \otimes_{R} G$. But then $\sum_{j<n}\left(\sum_{i<m}\left(d_{i}-a_{i}^{\prime}\right) r_{i j}\right) \otimes_{R} g_{j}=0$ in $D \otimes_{R} G$, whence $\sum_{i<m}\left(d_{i}-a_{i}^{\prime}\right) \otimes_{R} f_{i}=0$ in $D \otimes_{R} G$.

Since $D \in \mathcal{C}$, the map $i d_{D} \otimes_{R} \theta: D \otimes_{R} F \rightarrow D \otimes_{R} G$ is monic, so $\sum_{i<m}\left(d_{i}-a_{i}^{\prime}\right) \otimes_{R}$ $f_{i}=0$ in $D \otimes_{R} F$, in contradiction with $\sum_{i<m}\left(d_{i}+A\right) \otimes_{R} f_{i} \neq 0$ in $(D / A) \otimes_{R} F$.

An $\operatorname{AEC}(\mathcal{C}, \leq)$ is said to admit intersections if for each $C \in \mathcal{C}$ and each substructure $C^{\prime} \subseteq C$, the intersection of all strong substructures of $C$ containing $C^{\prime}$ is strong in $C$.

Corollary 1.5. Let $R$ be a ring and $\mathcal{D}$ be a class of modules of bounded injective dimension such that $\left({ }^{\perp} \mathcal{D}, \leq\right)$ is an AEC. If $\left({ }^{\perp} \mathcal{D}, \leq\right)$ admits intersections, then ${ }^{\perp} \mathcal{D}$ is a cotilting class; hence $\left({ }^{\perp} \mathcal{D}, \leq\right)$ has finite character.

Proof. Since $\left({ }^{\perp} \mathcal{D}, \leq\right)$ admits intersections, the class ${ }^{\perp} \mathcal{D}$ is closed under direct products by [3, Lemma 2.8]. Then ${ }^{\perp} \mathcal{D}$ is a cotilting class by [8, Theorem 4.3.23], and Theorem 1.4 applies.

By [3. Theorem 0.2(3)], all AECs of the form $\left({ }^{\perp} \mathcal{D}, \leq\right)$ over a Dedekind domain admit intersections, so we have

Corollary 1.6. Let $R$ be a Dedekind domain. Then each $A E C$ of the form $\left({ }^{\perp} \mathcal{D}, \leq\right)$ for a class of modules $\mathcal{D}$ has finite character.

There is also a positive answer in the hereditary artinian case:

Corollary 1.7. Let $R$ be a right hereditary and right artinian ring. Then each $A E C$ of the form $\left({ }^{\perp} \mathcal{D}, \leq\right)$ for a class of modules $\mathcal{D}$ has finite character.

Proof. Since $\mathcal{D}$ consists of modules of injective dimension $\leq 1$, 3, Lemmas 1.5 and 1.11] yield that ${ }^{\perp} \mathcal{D}$ is a torsion-free class of modules and ${ }^{\perp} \mathcal{D}$ is a cotilting class by [8, Theorem 4.3.23]. So again, $\left({ }^{\perp} \mathcal{D}, \leq\right)$ has finite character by Theorem 1.4. 


\section{AECS INDUCED BY TILTING MODULES}

We turn to the tilting setting and, more generally, to the setting of classes of finite type.

Given a class of modules $\mathcal{S}$, a module $M \in \operatorname{Mod}-R$ is called $\mathcal{S}$-filtered provided that $M$ contains a chain of submodules $\left(M_{\alpha} \mid \alpha \leq \sigma\right)$ such that $M_{0}=0, M_{\sigma}=M$, $M_{\alpha} \subseteq M_{\alpha+1}$ and $M_{\alpha+1} / M_{\alpha} \cong S_{\alpha}$ for some $S_{\alpha} \in \mathcal{S}$ for all $\alpha<\sigma$, and $M_{\alpha}=$ $\bigcup_{\beta<\alpha} M_{\beta}$ for all limit ordinals $\alpha \leq \sigma$. The chain $\left(M_{\alpha} \mid \alpha \leq \sigma\right)$ is called an $\mathcal{S}$-filtration of $M$.

A class of modules $\mathcal{C}$ is said to have refinements provided there is a cardinal $\kappa$ such that each module from $\mathcal{C}$ is $\mathcal{C}_{\kappa}$-filtered, where $\mathcal{C}_{\kappa}$ denotes the class of all $\leq \kappa$-generated modules in $\mathcal{C}$. This property is relevant here because of the following result proved in [3, $\S 1]$ :

Lemma 2.1. Let $R$ be a ring and $\mathcal{D} \subseteq \operatorname{Mod}-R$. Then $\left({ }^{\perp} \mathcal{D}, \leq\right)$ is an $\mathrm{AEC}$ if and only if the class ${ }^{\perp} \mathcal{D}$ is closed under direct limits and has refinements.

Remark 2.2. In view of Lemma 2.1 it is easy to see that our proof of Theorem 1.4 actually works in a more general setting for all AECs of the form $\left({ }^{\perp} \mathcal{D}, \leq\right)$ with ${ }^{\perp} \mathcal{D}$ closed under direct products and pure submodules. However, this more general setting still does not cover the tilting case as shown by the following example (which also shows that the reverse implication in Corollary 1.5 fails in general).

Example 2.3. Consider a ring $R$ which is right perfect but not left perfect (for instance, take $R=U T_{\omega}(K)$, the ring consisting of all upper tringular $\omega \times \omega$ matrices over a field $K$ which are constant on the main diagonal and have only finitely many non-zero entries above it; see [1, p. 322]). Let $\mathcal{D}$ denote a representative set of all simple right $R$-modules (for $R=U T_{\omega}(K)$, we can take $\mathcal{D}=\{K\}$ because the Jacobson radical $J$ of $R$ consists of the matrices that are zero on the main diagonal, and $R / J \cong K)$.

Then $\left({ }^{\perp} \mathcal{D}, \leq\right)$ is an AEC and ${ }^{\perp} \mathcal{D}$ is the class of all projective right $R$-modules (see [3, Example 2.11]). Since $R$ is not left perfect, ${ }^{\perp} \mathcal{D}$ is not closed under direct products by a classical result of Chase, and [3. Lemma 2.8] gives that $\left({ }^{\perp} \mathcal{D}, \leq\right)$ does not admit intersections. Clearly, ${ }^{\perp} \mathcal{D}={ }^{\perp} \mathcal{T}$ for the trivial tilting class $\mathcal{T}=$ Mod $-R$, so $\left({ }^{\perp} \mathcal{D}, \leq\right)$ has finite character by Theorem 2.4 (for $\left.\mathcal{S}=\{R\}\right)$.

Assume that $\mathcal{S} \subseteq \bmod -R$ is resolving. Let $\mathcal{D}=\mathcal{S}^{\perp}$ and $\mathcal{C}={ }^{\perp} \mathcal{D}$. Then $\mathcal{D}$ is of finite type and $\mathcal{C}$ coincides with the class of all direct summands of $\mathcal{S}$-filtered modules (see e.g. [8, Corollary 3.2.4]). This description of the class $\mathcal{C}$ makes it possible to prove yet another case of finite character:

Theorem 2.4. Let $R$ be a ring, $\mathcal{S}$ be a subclass of $\bmod -R$, and $\mathcal{C}={ }^{\perp}\left(\mathcal{S}^{\perp}\right)$. Assume that $(\mathcal{C}, \leq)$ is an $A E C$. Then $(\mathcal{C}, \leq)$ has finite character.

Proof. Possibly replacing $\mathcal{S}$ by ${ }^{\perp}\left(\mathcal{S}^{\perp}\right) \cap \bmod -R$, we can assume that $\mathcal{S}$ is resolving.

Consider an exact sequence $0 \rightarrow A \subseteq B \rightarrow M \rightarrow 0$ with $A, B \in \mathcal{C}$. Assume that for each finite subset $X$ of $A$ there is a monomorphism $f_{X}: A \rightarrow B$ with $f_{X} \uparrow X=\operatorname{id}_{X}$ and $f_{X}(A) \leq B$. By [8, Corollary 3.2.4], there is a module $C \in \mathcal{C}$ such that $A^{\prime}=A \oplus C$ is $\mathcal{S}$-filtered, and clearly $B^{\prime}=B \oplus C \in \mathcal{C}$. Extending $f_{X}$ to $C$ by identity, we can w.l.o.g. assume that $A=A^{\prime}$ is $\mathcal{S}$-filtered.

Fix an $\mathcal{S}$-filtration $\left(M_{\alpha} \mid \alpha \leq \sigma\right)$ of $A$. W.l.o.g., $\sigma$ is infinite. For each $\alpha<\sigma$, take a finitely generated submodule $A_{\alpha} \subseteq A$ such that $M_{\alpha+1}=M_{\alpha}+A_{\alpha}$. A subset 
$S \subseteq \sigma$ is said to be closed when $M_{\alpha} \cap A_{\alpha} \subseteq \sum_{\beta \in S, \beta<\alpha} A_{\beta}$ for each $\alpha \in S$. Consider the Hill family $\mathcal{F}=\left\{\sum_{\alpha \in S} A_{\alpha} \mid S\right.$ closed in $\left.\sigma\right\}$ (see [8, Theorem 4.2.6] for $\kappa=\omega$ ).

Let $F$ be a finite closed subset of $\sigma$ and $X$ be a finite $R$-generating subset of $A_{F}=\sum_{\alpha \in F} A_{\alpha}$. Then $A \cong f_{X}(A), A_{F}=f_{X}\left(A_{F}\right)$, and $B / f_{X}(A) \in \mathcal{C}$ by assumption. By [8, Theorem 4.2.6], $f_{X}(A) / A_{F}$ is $\mathcal{S}$-filtered, hence $B / A_{F} \in \mathcal{C}$. By [8. Theorem 4.2.6], $A$ is a directed union of the $A_{F}$ 's where $F$ runs over all finite closed subsets of $\sigma$, so $M \cong B / A$ is a direct limit of the $B / A_{F}$ 's. As $B / A_{F} \in \mathcal{C}$, also $M \in \mathcal{C}$ by [3, Lemma 1.5]; that is, $A$ is strong in $B$.

A module $M$ is $\sum$-pure split if every pure embedding $N^{\prime} \subseteq N$, where $N$ is a direct summand in a direct sum of copies of $M$, splits. For example, each $\sum$-pure injective module is $\sum$-pure split (see [ $\left.8,5.3\right]$ ).

Corollary 2.5. Let $R$ be a ring, $T$ be a tilting right $R$-module with the tilting class $\mathcal{T}=T^{\perp}$, and $\mathcal{C}={ }^{\perp} \mathcal{T}$. Then $(\mathcal{C}, \leq)$ is an $A E C$ if and only if $T$ is $\sum$-pure split. In this case $(\mathcal{C}, \leq)$ is of finite character.

Proof. By [6], $\mathcal{T}$ is of bounded type, so $\mathcal{T}=\mathcal{S}^{\perp}$ where $\mathcal{S}={ }^{\perp} \mathcal{T} \cap \bmod -R$ is resolving. Moreover, the class $\mathcal{C}$ has refinements by [8, Theorem 5.2.10], so Lemma 2.1 yields that $(\mathcal{C}, \leq)$ is an AEC iff $\mathcal{C}$ is closed under direct limits. But the latter is equivalent to $T$ being $\sum$-pure split by [8, Proposition 5.3.4]. The final claim follows by Theorem 2.4.

\section{REFERENCES}

[1] F. W. Anderson and K. R. Fuller, Rings and Categories of Modules, 2nd ed., GTM 13, Springer-Verlag, New York, 1992. MR.1245487(94i:16001)

[2] J. Baldwin, Categoricity, www.math.uic.edu/jübaldwin.

[3] J. T. Baldwin, P. C. Eklof, J. Trlifaj, ${ }^{\perp} N$ as an abstract elementary class, Annals of Pure Appl. Logic 149(2007), 25-39. MR2364195

[4] S. Bazzoni, Cotilting and tilting modules over Prüfer domains, Forum Math. 19(2007), 10051027. MR2367952

[5] S. Bazzoni, When are definable classes tilting and cotilting classes?, to appear in J. Algebra.

[6] S. Bazzoni, J. Šťovíček, All tilting modules are of finite type, Proc. Amer. Math. Soc. 135(2007), 3771-3781. MR.2341926

[7] W. Crawley-Boevey, Infinite-dimensional modules in the representation theory of finitedimensional algebras, CMS Conf. Proc. 23, Amer. Math. Soc., Providence, RI, 1998, 2954. MR:1648602 (99m:16016)

[8] R. Göbel and J. Trlifaj, Approximations and Endomorphism Algebras of Modules, GEM 41, W. de Gruyter, Berlin, 2006. MR2251271 (2007m:16007)

[9] T. Hyttinen and M. Kesälä, Independence in finitary abstract elementary classes, Annals of Pure Appl. Logic 143(2006), 103-138. MR2258625 (2007k:03087)

[10] D. W. Kueker, Abstract Elementary Classes and Infinitary Logics, preprint (2008).

[11] M. Prest, Model Theory and Modules, LMS Lecture Note Ser. 130, Cambridge Univ. Press, Cambridge, 1988. MR 933092 (89h:03061)

[12] S. Shelah, Classification of nonelementary classes, II. Abstract elementary classes, In J. T. Baldwin, ed., Classification Theory (Chicago, IL, 1985), Lecture Notes in Math. 1292, Springer, Berlin, 1987, 419-497. MR.1033034 (91h:03046)

[13] B. Stenström, Rings of Quotients, Springer-Verlag, New York-Heidelberg, 1975. MR0389953 $(52: 10782)$

[14] J. Stovíček, All n-cotilting modules are pure-injective, Proc. Amer. Math. Soc. 134(2006), 1891-1897. MR2215116(2007a:16005)

Faculty of Mathematics and Physics, Department of Algebra, Charles University, Sokolovská 83, 18675 Prague 8, Czech Republic

E-mail address: trlifaj@karlin.mff.cuni.cz 